\title{
Gratis Open Access
}

Peter Suber

\section{Source}

https:/llegacy.earlham.edu/ peters/fos/overview.htm Peter Suber.

Gratis OA removes price barriers alone. [...] Gratis OA is free of charge, but not free of copyright of licensing restrictions. Users must either limit themselves to fair use or seek permission to exceed it 